\title{
Cross-Cultural and Gender Comparison of Emotional Connotation of Words
}

\author{
Yaxuan Liang ${ }^{1}$ and Xiaohong $\mathrm{Xu}^{\#}$ \\ ${ }^{1}$ Arcadia High School, Arcadia, CA, USA \\ \#Advisor
}

\section{$\underline{\text { ABSTRACT }}$}

So far, scientists have researched to explore the emotional connotation of words in different native languages and genders. This study investigates how connotations of words differ by cultures and genders by inspecting valence values, which indicates how happy people feel about specific words in datasets obtained from USA, Spain, and Portuguese. To fulfill the objective, we categorized people's feelings evoked by the words based on a criterion created by adding or subtracting standard deviation to or from valence means for the upper and lower bound. Then, words with valence values outside of the upper and lower bounds were categorized as emotional words (positive or negative) and were analyzed. The results show that people of different cultures are more connected regarding the perception of negative connotation of words than positive connotation. Moreover, Portuguese and Spanish are more connected than either of them with the US as they have more emotion words in common. On the gender aspect, we notice that females tend to give more extreme ratings for words than males. Furthermore, the analysis shows that males have a more positive feeling towards sex-related words than females. Overall, this study outlines a way for people interested in anthropology to understand the differences in the emotional connotation of words between males and females and across cultures.

\section{Introduction}

People have longed to understand the emotional connotation of the words. Setting this as an aim, ANEW(the Affective Norms for English Words) examined the emotional connotation of 1034 English words using three variables - "valence", "arousal", and "dominance"(Bradley \& Lang, 1999). "Valence" represents how happy or unhappy the word makes subjects of the survey feel; "arousal" describes the extent of excitement, while "dominance" refers to the degree of feeling of control. The original ANEW study found that the "valence" value had a quadratic relationship with the "arousal" value. In other words, the "arousal" value increases as "valence" values reach two extremes of the spectrum. On the other hand, the "dominance" value has a linear relationship with the "valence" value, meaning that the "dominance" value increases as the "valence" value increases.

Since then, many researchers from different countries and cultural backgrounds have conducted studies similar to ANEW to explore the emotional connotation of words in different native languages and genders( Montefinese et al., 2013; Redondo et al., 2007; Soares et al., 2011). These rich datasets allow us to perform inter-comparison between studies to probe the effects of culture and gender on the emotional connotation of words. In particular, studies in Spanish (Redondo et al., 2007), Portuguese (Soares et al., 2011), and the original English ANEW study (Bradley \& Lang, 1999) are based on the same word list and provide both male and female data. Such a unified word list allows for more accurate inter-comparison.

In the current study, we wish to understand how emotional connotations of words may differ across cultures and genders by examining values published in these three studies. The idea is that cultures may impact interpretation of words. Additionally, there might be inherent gender differences in the emotional connotation of words that show 
cross-culture consistency. Since Spain and Portuguese are graphically and culturally closer to each other than to America, we predict that emotional values from Spanish and Portuguese datasets will be more closely related than they are with the English dataset. The current study's findings may help elucidate subtle grains of differences in the emotional connotation of words affected by culture and gender differences.

\section{Methods}

In addition to variables like "valence" and "arousal" in the original ANEW study, the Portuguese and Spanish studies also included other lexico-semantic variables, including "concreteness", "imageability", "context availability", "familiarity", "frequency", and "length". Some other studies have used these variables to examine how semantic variables may impact emotional word processing(Yao et al., 2016). However, in the current research, only "valence" value is used to analyze the perspectives of people from different cultures since our research focuses on how words directly appeal to subjects' emotions rather than some other aspects that affect the processing of affective words (eg. concreteness, imageability).

First, "valence" means for the word list from each study was gathered in one spreadsheet. The standard deviation of the "valence" mean and mean of the "valence" mean are calculated using the "STDEV" and "AVERAGE" functions in Excel. We added or subtracted the average by the standard deviation to get the upper and lower critical values, respectively. If one word's "valence" mean value is higher than the upper critical value, the word carries a positive emotional connotation. If the value is lower than the lower critical value, the word carries a negative emotional connotation. We consider both negative and positive words as emotional words. Otherwise, if a word's valence value falls between the upper and lower critical values, then the word is categorized as non-emotional.

After this process, we assign each word a representation value, which can be "1", "-1", or " 0 " by using the IF function. Specifically, positive emotional words receive the value " 1 ", and negative words the value "-1", whereas non-emotional words the value " 0 ".

We then put the representation values, valence mean values, and words from different languages into one spreadsheet and analyzed the similarities and differences across the three datasets to evaluate how each culture and gender process each word.

\section{Results}

\section{More negative than positive}

By examining the dataset, we found that people in all three cultures rated more words as "negative" than "positive" (Table I). Moreover, the words rated as negative by all three cultures accounted for $71.8 \%, 68.7 \%$, and $65.0 \%$ of the total number of negative words for the English, Portuguese and Spanish datasets, respectively. By comparison, words rated as positive accounted for $58.5 \%, 58.2 \%$, and $55.5 \%$ of the total number of positive words for the English, Portuguese and Spanish datasets, respectively. We noticed that the ratio of shared emotional words was higher for the negative category than the positive category (paired t-test, $\mathrm{p}=0.01$ ). This result suggests that people in different cultures may be more connected regarding the perception of negative connotation of words since more negatively connotated words are shared among cultures than positively connotated words. Accordingly, people's perspectives on negative words may be more stable and less susceptible to cultural modifications than positive words, which show more variability across cultures. 


\begin{tabular}{|l|l|l|l|l|}
\hline & $\begin{array}{l}\text { Total number of } \\
\text { words that are } \\
\text { rated as positive }\end{array}$ & $\begin{array}{l}\text { Total number of } \\
\text { words that are rated } \\
\text { as negative }\end{array}$ & $\begin{array}{l}\text { Numbers of words that } \\
\text { are rated as positive by } \\
\text { all three groups of people }\end{array}$ & $\begin{array}{l}\text { Numbers of words that } \\
\text { are rated as negative by } \\
\text { all three groups of people }\end{array}$ \\
\hline English & 207 & 241 & 121 & 173 \\
\hline Portuguese & 208 & 252 & 121 & 173 \\
\hline Spanish & 218 & 266 & 121 & 173 \\
\hline
\end{tabular}

Table I. The number of total and shared emotional words across all three cultures.

\section{Closer relationship between Portuguese and Spanish}

Next, we performed pairwise analysis of shared positive and negative words in the three samples (Spanish-Portuguese, Portuguese-English and Spanish-English). As shown in Table II, the ratios of shared positive words between SpanishPortuguese pair is higher than the ratios of shared positive words for the Portuguese-English and Spanish-English pairs. At the same time, the ratio of shared negative words between the Spanish-Portuguese pair is also higher than the ratios of shared negative words for the Portuguese-English and Spanish-English pairs, as shown in Table III. From pattern and ratio values exhibited in Table II and Table III, we can conclude that there is significantly more similarity in emotional connotation of words between Spanish and Portuguese than either one with the English (t-test, $\mathrm{p}=0.06$ ).

\begin{tabular}{|l|l|l|l|}
\hline & $\begin{array}{l}\text { Ratio of shared posi- } \\
\text { tive words between } \\
\text { two cultures over total } \\
\text { positive words for } \\
\text { Spanish }\end{array}$ & $\begin{array}{l}\text { Ratio of shared posi- } \\
\text { tive words between } \\
\text { two cultures over total } \\
\text { positive words for Por- } \\
\text { tuguese }\end{array}$ & $\begin{array}{l}\text { Ratio of } \\
\text { shared positive words } \\
\text { between two cultures } \\
\text { over total positive } \\
\text { words for English }\end{array}$ \\
\hline Spanish \& Portuguese & $76.6 \%$ & $80.3 \%$ & N/A \\
\hline Spanish \& English & $64.7 \%$ & N/A & $68.1 \%$ \\
\hline English \& Portuguese & N/A & $69.2 \%$ & $69.6 \%$ \\
\hline
\end{tabular}

Table II. Ratios of shared positive words in pair-wise comparison in the three cultures.

\begin{tabular}{|l|l|l|l|}
\hline & $\begin{array}{l}\text { Ratio of negative } \\
\text { words between two } \\
\text { cultures over total neg- } \\
\text { ative words for Span- } \\
\text { ish }\end{array}$ & $\begin{array}{l}\text { ratio of negative words } \\
\text { between two cultures } \\
\text { over total negative } \\
\text { words for Portuguese }\end{array}$ & $\begin{array}{l}\text { ratio of negative } \\
\text { cultures otween two } \\
\text { negative words for } \\
\text { English }\end{array}$ \\
\hline Spanish \& Portuguese & $79.7 \%$ & $84.1 \%$ & N/A \\
\hline Spanish \& English & $75.2 \%$ & N/A & $83.0 \%$ \\
\hline English \& Portuguese & N/A & $75.8 \%$ & $79.3 \%$ \\
\hline
\end{tabular}


Table III. Ratios of shared negative words in pair-wise comparison in the three cultures.

\section{Comparing words ratings between the two genders across cultures}

Previously, some studies were done to compare the emotional connotation of words in males or females of the same culture. One founding is that women tend to be more emotional since "women rate positive things as more pleasant and negative things as less pleasant than men "(Montefinese et al., 2013). To examine whether this finding is true across different cultures, we calculated the mean of valence values provided by the two genders in English, Portuguese and Spanish datasets separately. Noticing the gender variability in ratings, we set an upper bound and lower bound to define emotional words using the mean valence value and standard deviation of either gender (Table IV). Upon examining these parameters, we noted that the mean valence value for all 1034 words does not differ consistently between females and males of all three cultures. However, the standard deviation is consistently higher in females than males, demonstrating higher variability in ratings in females. As expected, the upper and lower bounds for determining emotional words are more extreme in females than males, demonstrating that females of all three cultures rated positive words more positive and negative words more negative than males (Table V).

\begin{tabular}{|l|l|l|l|l|}
\hline & Female Valence Mean & Male Valence Mean & $\begin{array}{l}\text { Female Valence Stand- } \\
\text { ard Deviation }\end{array}$ & $\begin{array}{l}\text { Male Valence Stand- } \\
\text { ard Deviation }\end{array}$ \\
\hline English & 5.08 & 5.24 & 2.18 & 1.79 \\
\hline Spanish & 4.74 & 4.74 & 2.17 & 2.11 \\
\hline Portuguese & 4.98 & 5.07 & 1.91 & 1.69 \\
\hline
\end{tabular}

Table IV. Mean and standard deviation of valence value of all 1034 words for males and females of each culture

\begin{tabular}{|l|l|l|l|l|}
\hline & $\begin{array}{l}\text { Female Valence Upper } \\
\text { Bound }\end{array}$ & $\begin{array}{l}\text { Male Valence Upper } \\
\text { Bound }\end{array}$ & $\begin{array}{l}\text { Female Valence Lower } \\
\text { Bound }\end{array}$ & $\begin{array}{l}\text { Male Valence Lower } \\
\text { Bound }\end{array}$ \\
\hline English & 7.26 & 7.03 & 2.90 & 3.45 \\
\hline Spanish & 6.91 & 6.85 & 2.57 & 2.64 \\
\hline Portuguese & 6.88 & 6.76 & 3.07 & 3.38 \\
\hline
\end{tabular}

Table V. Upper and lower bound of valence value used to determine emotional words for males and females of each culture.

\section{Two Genders Perspectives on Sex-related Words}

Interestingly, previous studies also note one peculiar fact -sexual words are more emotionally arousal for males than females(Montefinese et al., 2013). Here, by applying the upper bound and lower bound for identifying and categorizing the affective words, we found that across three cultures, more words are rated as positive in females than males (Table VI). Despite this, it seems that more sexual words are rated as positive by males in all three cultures than females (Figure 1 and 2). For example, females in all three cultures rate words related to female sex organs as neutral. On the other hand, males in all three cultures gave much higher valence ratings to these terms than females. Together these results point to a consistent sex difference in the emotional connotation of words regarding a particular categorysexual words. 


\begin{tabular}{|l|l|l|}
\hline & $\begin{array}{l}\text { Total number of positive words rated } \\
\text { by female }\end{array}$ & $\begin{array}{l}\text { Total number of positive words rated } \\
\text { by male }\end{array}$ \\
\hline English & 222 & 191 \\
\hline Spanish & 215 & 207 \\
\hline Portuguese & 213 & 197 \\
\hline
\end{tabular}

Table VI. Total number of positive words rated by either gender across three cultures

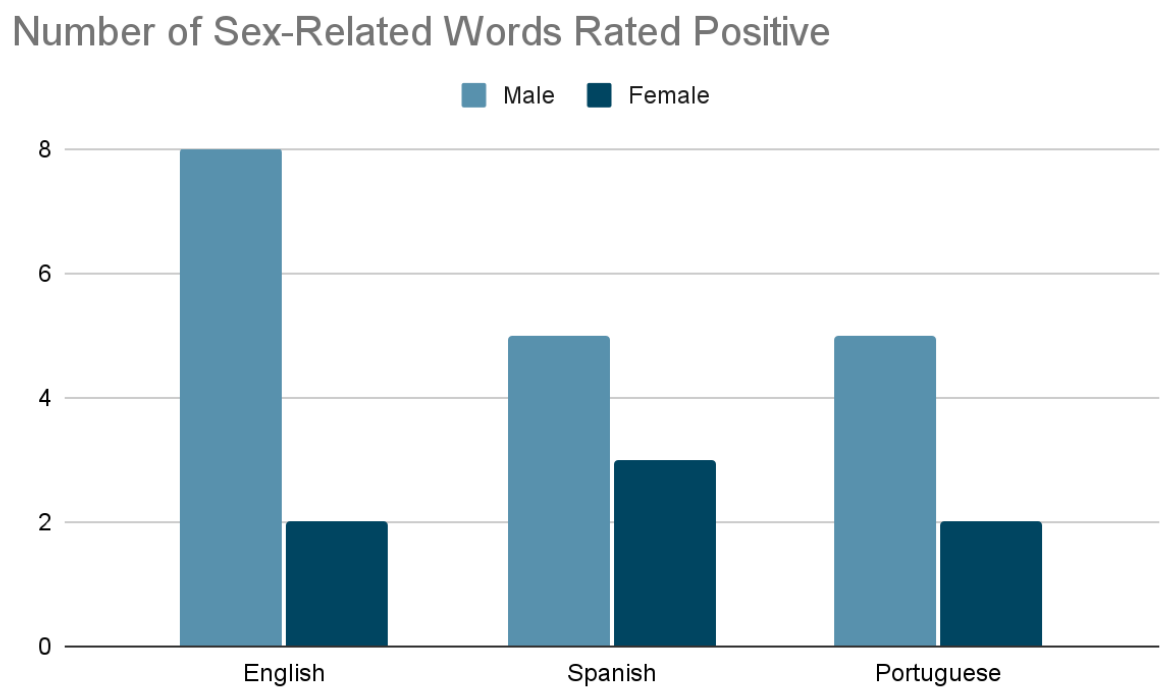

Figure 1. Total number of sexually words rated positively by either gender across three cultures

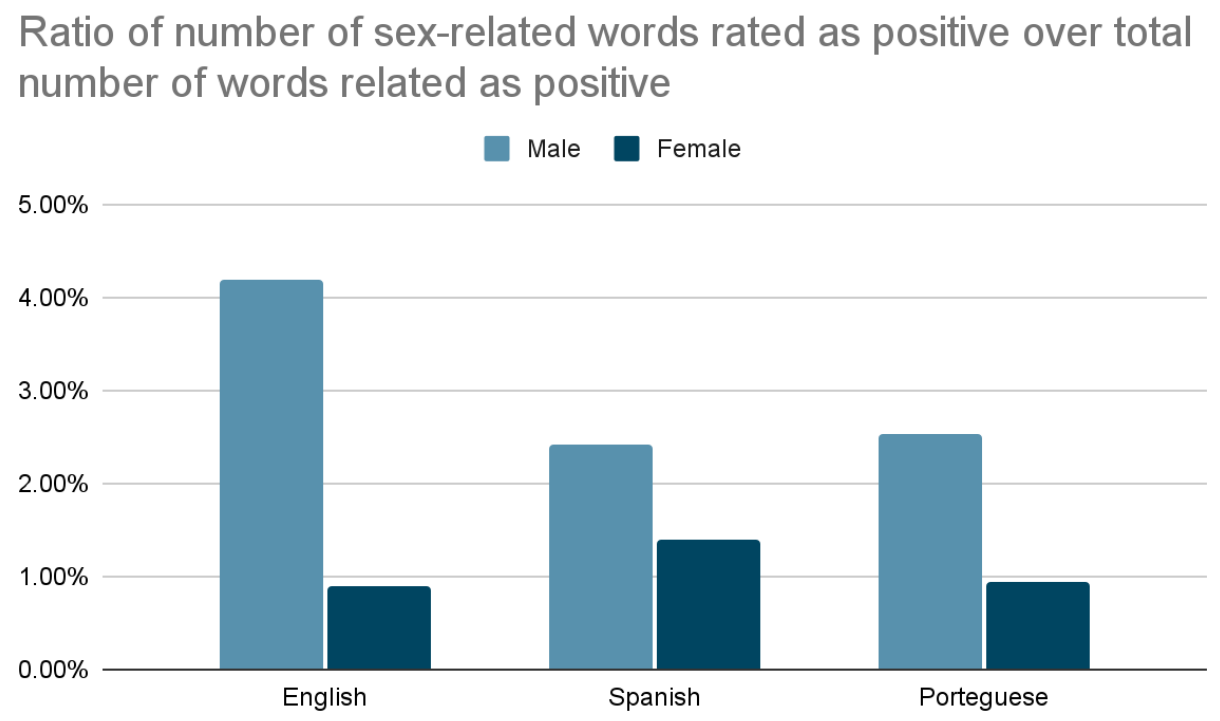

Figure 2. Ration of sexually words rated positively by either gender across three cultures 


\section{Two Genders Perspectives on Nature-related Words}

Interestingly, previous studies have suggested that females give higher ratings for nature-related words than males (Montefinese et al., 2013). Using the same method for finding two genders' perspectives on sexual words, we found that females rate more nature-related words as positive than males do in all three cultures. However, an intriguing observation is that such sex differences are more diminutive in the Spain and Portuguese datasets than in the American dataset. Moreover, because females rated more words as positive than males, when we calculate the ratio of naturalrelated positive words divided by the total number of positive words, these sex differences further diminish. Together these results do not support a substantial sex difference in the emotional connotation of nature-related words.

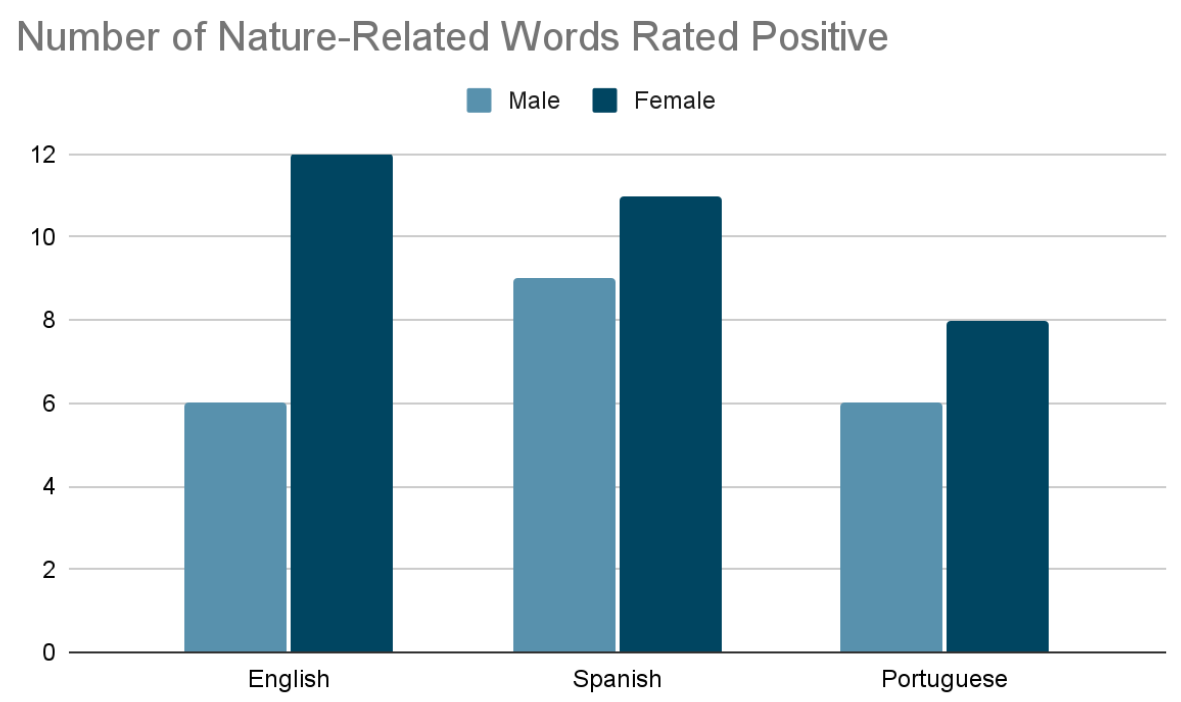

Figure III. Total number of nature-related words rated positively by either gender across three cultures

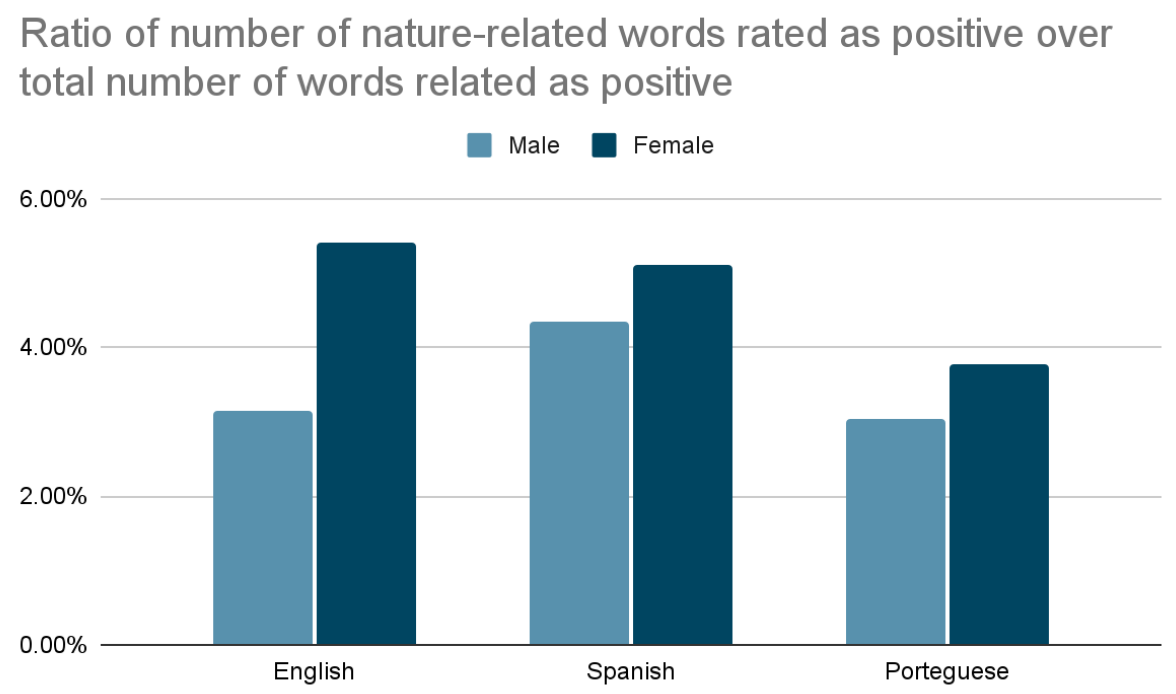

Figure IV. Ratio of nature-related words rated positively by either gender across three cultures 


\section{Discussion}

This study found that the ratios of the total number of negative words and the number of shared negative words in all three cultures are higher than those of the positive words. Therefore, people in different cultures are more connected regarding the perception of negative words since more connotations of negative words are shared among cultures than positive ones. Accordingly, people's perspectives on negative words are more conserved since they vary less across cultures than positive words' connotations.

Investigation in the relationships between three countries where the data were collected was also conducted in this research. As predicted at the very beginning of this study, Portuguese and Spain have a closer relationship than their relationships with America in the emotional connotation of words as their have more positive and negative words in common. It seems that their geographical closeness can explain the intimacy between Spain and Portugal because it is supported in one study done by Rebecca A. Martusewicz that geographical features, which determine the availability of resources, are essential factors that influence the culture(Johnson \& Martusewicz, n.d.).

Regarding the valence ratings of words provided by two genders, we noticed that females in all three cultures have a higher upper bound and a lower bound to classify whether the word is affective than men do. Therefore, we can conclude that females tend to rate words with a more extreme value than men. This finding suggests that the patterns observed in Italian adaptation of ANEW - women tend to rate both pleasant and unpleasant words with more hedonic values than men - can be applied to other cultures because of the consistency of the observation.

Furthermore, we also found that men in all three cultures rate sex-related words with more positive values than females. Given that females tend to rate words with more extreme values, we can conclude that males generally feel more positive about female sexual organs than females do. These observations expand the range that pattern found in Italian adaptation of ANEW, which only shows the differences in perspectives between males and females in Italy, can be applied.

Upon the inspection of the ratings of nature-related words provided by participants in different cultures, we found that the differences between the perspectives of two genders in Spain and Portuguese are very similar. Therefore, it supports an idea mentioned in the previous findings and the previous parts of this study that Spain has a closer relationship with Portuguese than any of them do with America. Moreover, using upper bounds precisely calculated for two genders, we discovered that females rate more nature-related terms with positive values than men. However, such sex difference is much less pronounced than the male bias in rating sex-related words.

\section{Conclusion}

To conclude, this study is based on the data gathered from the research of emotional connotation of words conducted in different cultures. The purpose of this research is to explore the innate interpretation of words, which can be reflected by consistent gender differences in affective ratings, and the possible relationships between different cultures by examining how affective ratings of the same set of terms differ across cultures and genders. In the end, the analysis of data points out that, as we predicted at the beginning of this research, Portuguese and Spain indeed have closer relationships than relationships between any of them and America. Furthermore, the patterns found in the Italian adaptation of ANEW regarding the gender differences are confirmed in all three cultures examined in this study.

After all, this study can contribute to future research exploring gender differences in perceiving different types of words and relationships between countries. The findings of this research help scholars to understand the consistent gender differences across cultures and the closeness of Spain and Portuguese. 


\section{Acknowledgments}

I thank Xiaohong $\mathrm{Xu}$ for mentorship and guiding me through all the processes. I thank Viax Institution for teaching me the format of a research paper and providing mental support.

\section{References}

Bradley, M. M., \& Lang, P. J. (n.d.). Affective Norms for English Words (ANEW): Instruction Manual and Affective Ratings. 49.

Johnson, L., \& Martusewicz, R. (n.d.). EcoJustice Education. Retrieved August 12, 2021, from https://www.academia.edu/29669427/EcoJustice_Education

Montefinese, M., Ambrosini, E., Fairfield, B., \& Mammarella, N. (2013). The adaptation of the Affective Norms for English Words (ANEW) for Italian. Behavior Research Methods, 46. https://doi.org/10.3758/s13428-013-0405-3

Redondo, J., Fraga, I., Padron, I., \& Comesaña, M. (2007). The Spanish adaptation of ANEW (Affective Norms for English Words). Behavior Research Methods, 39, 600-605. https://doi.org/10.3758/BF03193031

Soares, A., Comesaña, M., Pinheiro, A., Simões, A., \& Frade, S. (2011). The adaptation of the Affective Norms for English Words (ANEW) for European Portuguese. Behavior Research Methods, 44, 256-269.

https://doi.org/10.3758/s13428-011-0131-7

Yao, Z., Wu, J., Zhang, Y., \& Wang, Z. (2016). Norms of valence, arousal, concreteness, familiarity, imageability, and context availability for 1,100 Chinese words. Behavior Research Methods, 49. https://doi.org/10.3758/s13428-

016-0793-2 